\title{
T-Stability of the Heun Method and Balanced Method for Solving Stochastic Differential Delay Equations
}

\author{
Xiaolin Zhu and Hu Peng \\ School of Mathematics, Hefei University of Technology, Hefei 230009, China \\ Correspondence should be addressed to Xiaolin Zhu; zxl_hfut@126.com \\ Received 9 March 2014; Accepted 15 May 2014; Published 11 June 2014 \\ Academic Editor: Senlin Guo
}

Copyright ( 2014 X. Zhu and H. Peng. This is an open access article distributed under the Creative Commons Attribution License, which permits unrestricted use, distribution, and reproduction in any medium, provided the original work is properly cited.

\begin{abstract}
This paper studies the T-stability of the Heun method and balanced method for solving stochastic differential delay equations (SDDEs). Two T-stable conditions of the Heun method are obtained for two kinds of linear SDDEs. Moreover, two conditions under which the balanced method is T-stable are obtained for two kinds of linear SDDEs. Some numerical examples verify the theoretical results proposed.
\end{abstract}

\section{Introduction}

Stochastic differential delay equations (SDDEs) are the promotion of stochastic differential equations (SDEs) and differential delay equations (DDEs). These kinds of equations consider not only the stochastic factors in the process of the development of a system, but also the impact of the delay. As an important mathematical model, SDDEs have been applied widely in many areas, such as stochastic control, economics, and biology. Since it is difficult to find the analytic solutions to SDDEs, to get the numerical solutions to SDDEs generated by some numerical methods is commonly used. For a numerical method, it is important to analyze its stability.

The general form of SDDEs with Gaussian white noise is

$$
\begin{aligned}
& \mathrm{d} X(t)=f(t, X(t), X(t-\tau)) \mathrm{d} t \\
& \quad+g(t, X(t), X(t-\tau)) \mathrm{d} W(t), \quad t \in[0, T], \\
& X(t)=\varphi(t), \quad t \in[-\tau, 0],
\end{aligned}
$$

where $\tau>0, \varphi(t) \in \mathbf{C}\left([-\tau, 0], \mathbf{R}^{m}\right), f: \mathbf{R}^{+} \times \mathbf{R}^{m} \times \mathbf{R}^{m} \rightarrow$ $\mathbf{R}^{m}, g: \mathbf{R}^{+} \times \mathbf{R}^{m} \times \mathbf{R}^{m} \rightarrow \mathbf{R}^{m \times d}$, and $W(t)$ is a standard $d$-dimensional Wiener process. Equation (1) has a unique solution if $f$ and $g$ are sufficiently smooth and satisfy the following conditions:

$$
\begin{aligned}
\left|f\left(t, x_{1}, y_{1}\right)-f\left(t, x_{2}, y_{2}\right)\right| & \vee\left|g\left(t, x_{1}, y_{1}\right)-g\left(t, x_{2}, y_{2}\right)\right| \\
\leq L\left(\left|x_{1}-x_{2}\right|+\left|y_{1}-y_{2}\right|\right) & \\
|f(t, x, y)|^{2} \vee|g(t, x, y)|^{2} & \leq K\left(1+|x|^{2}+|y|^{2}\right)
\end{aligned}
$$

where $t \geq 0, x, y, x_{1}, y_{1}, x_{2}, y_{2} \in \mathbf{R}^{m}$, and $L$ and $K$ are constants. The condition in (2) is called Lipschitz condition, and the condition in (3) is called the linear growth condition.

The main numerical methods for SDDEs are EulerMaruyama method [1,2] and Milstein method [3] at present. The mean square stability of these methods for SDDEs has been well studied. Cao et al. [1] studied the mean square stability of Euler-Maruyama method for linear SDDEs. Liu et al. [2] studied the mean square stability of the semiimplicit Euler method for linear SDDEs. Wang and Zhang [3] discussed the mean square stability of Milstein method for linear SDDEs. Wang and Chen $[4,5]$ studied the mean square 
stability of semi-implicit Euler method for nonlinear neutral SDDEs and that of Heun methods for nonlinear SDDEs. Tan et al. [6] discussed the mean square stability of balanced methods for SDDEs. T-stability is introduced by Saito et al. in [7-9], and it is another kind of stability with respect to the approximate sequence of sample path. Cao [10] studied the T-stability of the semi-implicit Euler method for delay differential equations with multiplicative noise. Rathinasamy and Balachandran [11] studied the T-stability of the split-step $\theta$-methods for linear SDDEs. Yang and Liu [12] discussed the T-stability of the $\theta$-method for a stochastic pantograph differential equation.

Applying the Heun method [5] to (1) gives

$$
\begin{aligned}
& X_{n+1} \\
& \qquad X_{n}+\frac{1}{2}\left[f\left(t_{n}, X_{n}, X_{n-m}\right)\right. \\
& \left.\quad+f\left(t_{n+1}, X_{n}+h f\left(t_{n}, X_{n}, X_{n-m}\right), X_{n-m+1}\right)\right] h \\
& \quad+g\left(t_{n}, X_{n}, X_{n-m}\right) \Delta W_{n},
\end{aligned}
$$

where $h>0$ is a step size with $\tau=m h$ for a positive integer $m$ and $t_{n}=n h . X_{n}$ is an approximation of $X\left(t_{n}\right)$, and $X_{n}=\varphi\left(t_{n}\right)$ if $t_{n} \leq 0 . \Delta W_{n}=W\left(t_{n+1}\right)-W\left(t_{n}\right) \sim N(0, h)$.

The mean square stability of the Heun method in (4) was studied in [5], but there is no result about T-stability of the method at present. This paper gives two T-stable conditions of the Heun method (4) for two kinds of linear SDDEs.

The balanced method for solving (1) is

$$
\begin{aligned}
X_{n+1}= & X_{n}+f\left(t_{n}, X_{n}, X_{n-m}\right) h+g\left(t_{n}, X_{n}, X_{n-m}\right) \Delta W_{n} \\
& +C\left(X_{n}, X_{n-m}\right)\left(X_{n}-X_{n+1}\right),
\end{aligned}
$$

where $C\left(X_{n}, X_{n-m}\right)=C_{0}\left(X_{n}, X_{n-m}\right) h+C_{1}\left(X_{n}, X_{n-m}\right)\left|\Delta W_{n}\right|$ and $C_{0}, C_{1}$ are $d \times d$ real matrix functions.

Let $M(x, y)=I+\beta_{0} C_{0}(x, y)+\beta_{1} C_{1}(x, y)$, where $I$ is a unit matrix, $\beta_{0} \in[0, \alpha], \alpha \geq h, \beta_{1} \geq 0$, and $(x, y) \in R^{d} \times R^{d}$. Assume that $M(x, y)$ is invertible with $\left|(M(x, y))^{-1}\right| \leq K<$ $\infty$.

The mean square stability of the balanced method for SDEs and SDEs with jumps was studied in $[13,14]$, respectively. In 2011, Tan et al. [6] applied the balanced method to SDDEs and discussed the mean square convergence and stability of this method. However, there is no research result about T-stability of the balanced method (5) at present. In this paper, the conditions under which the balanced method (5) is T-stable are obtained for two kinds of linear SDDEs.

Section 2 introduces the stochastically asymptotically stable conditions in the large for two kinds of linear SDDEs. In Section 3, T-stability of the Heun method equipped with a specified driving process is discussed and the corresponding step size range is given. Section 4 studies T-stability of the balanced method, and Section 5 uses some numerical examples to verify the results given in this paper.

\section{Asymptotic Stability of Analytical Solution}

Consider the following two scalar linear test equations:

$$
\begin{gathered}
\mathrm{d} X(t)=[a X(t)+b X(t-\tau)] \mathrm{d} t \\
+c X(t) \mathrm{d} W(t), \quad t \in[0, T] \\
X(t)=\varphi(t), \quad t \in[-\tau, 0], \\
\mathrm{d} X(t)=[a X(t)+b X(t-\tau)] \mathrm{d} t \\
+[c X(t)+d X(t-\tau)] \mathrm{d} W(t), \quad t \in[0, T] \\
X(t)=\varphi(t), \quad t \in[-\tau, 0] .
\end{gathered}
$$

Let $\left(\Omega, \mathbf{F},\left\{F_{t}\right\}_{t \geq 0}, P\right)$ be a complete probability space with a filtration $\left\{F_{t}\right\}_{t \geq 0}$, which is right continuous, and each $F_{t}$ contains all $P$-null sets in F. In (6) and (7), $a, b, c, d \in$ $\mathbf{R} ; \tau>0 ; W(t)$ is a one-dimensional standard Wiener process; initial function $\varphi(t) \in \mathbf{C}\left([-\tau, 0], \mathbf{R}^{m}\right)$ is $F_{0}$-measurable and $E\|\varphi\|^{2}<\infty$. Equations (6) and (7) have unique strong solution if (6) and (7) meet Lipschitz condition in (2) and the linear growth condition in (3).

Definition 1 (see [10]). The solution of (1) is stochastically asymptotically stable in the large if

$$
P\left(\lim _{t \rightarrow \infty}|X(t, \varphi)|=0\right)=1
$$

for all initial functions $\varphi$.

From Corollary 3.2 in [15], we get Lemmas 2 and 3 as follows.

Lemma 2. The solution of (6) is stochastically asymptotically stable in the large if parameters $a, b$, and $c$ in (6) satisfy

$$
a<-|b|-\frac{1}{2} c^{2}
$$

Lemma 3. The solution of (7) is stochastically asymptotically stable in the large if parameters $a, b, c$, and $d$ in (7) satisfy

$$
a<-|b|-\frac{1}{2}(|c|+|d|)^{2} .
$$

\section{T-Stability of the Heun Method}

Definition 4 (see [10]). Suppose that the condition in (9) or in (10) is fulfilled. A numerical scheme equipped with a specified driving process is said to be T-stable if

$$
\lim _{n \rightarrow \infty}\left|X_{n}\right| \longrightarrow 0 \quad \text { a.s. }
$$

for the driving process, where $X_{n}$ is the numerical solution generated by the numerical scheme applied to the test equation (6) or (7).

For analyzing T-stability, we focus our attention on the trajectory of numerical solution. A specified driving 
process proposed in Definition 4 is used to approximate the Wiener increment $\Delta W_{n}$ of the numerical methods. This paper analyzes the Heun method equipped with two-point random variables for the driving process. So $\Delta W_{n}:=\eta_{n} \sqrt{h}$ and $P\left(\eta_{n}=\right.$ $\pm 1)=1 / 2$, where $P$ denotes the probability.

The Heun method applied to (6) and (7), respectively, gives

$$
\begin{aligned}
X_{n+1}= & \left(1+a h+\frac{1}{2} a^{2} h^{2}+c \Delta W_{n}\right) X_{n} \\
& +\frac{1}{2} b h(1+a h) X_{n-m}+\frac{1}{2} b h X_{n-m+1}, \\
X_{n+1}= & \left(1+a h+\frac{1}{2} a^{2} h^{2}+c \Delta W_{n}\right) X_{n} \\
& +\left[\frac{1}{2} b h(1+a h)+d \Delta W_{n}\right] X_{n-m}+\frac{1}{2} b h X_{n-m+1},
\end{aligned}
$$

where $h>0$ is a step size with $\tau=m h$ for a positive integer $m$ and $t_{n}=n h, X_{n} \approx X\left(t_{n}\right)$ if $t_{n} \leq 0, X_{n}=\varphi\left(t_{n}\right)$.

For the Heun method in (12), we have

$$
\begin{aligned}
\left|X_{n+1}\right| \leq & \left(\left|1+a h+\frac{1}{2} a^{2} h^{2}+c \Delta W_{n}\right|\right. \\
& \left.+\left|\frac{1}{2} b h(1+a h)\right|+\left|\frac{1}{2} b h\right|\right) \\
& \times \max \left\{\left|X_{n}\right|,\left|X_{n-m}\right|,\left|X_{n-m+1}\right|\right\} \\
= & \left(\left|\frac{1}{2}(1+a h)^{2}+\frac{1}{2}+c \eta_{n} \sqrt{h}\right|\right. \\
& \left.+\left|\frac{1}{2} b h(1+a h)\right|+\left|\frac{1}{2} b h\right|\right) \\
& \times \max \left\{\left|X_{n}\right|,\left|X_{n-m}\right|,\left|X_{n-m+1}\right|\right\} .
\end{aligned}
$$

Let

$$
\begin{aligned}
R(h, a, b, c)= & \left|\frac{1}{2} b h(1+a h)\right|+\left|\frac{1}{2} b h\right| \\
& +\left|\frac{1}{2}(1+a h)^{2}+\frac{1}{2}+c \eta_{n} \sqrt{h}\right| .
\end{aligned}
$$

It is clear that $\left|X_{n}\right| \rightarrow 0$, a.s. $(n \rightarrow \infty)$ if $P(R(h, a, b, c)<$ $1)=1$, and therefore the Heun method in (12) is T-stable. Denote

$$
\begin{aligned}
R_{T}(h, a, b, c) & \\
= & \left(\left|\frac{1}{2} b h(1+a h)\right|+\left|\frac{1}{2} b h\right|\right. \\
& \left.+\left|\frac{1}{2}(1+a h)^{2}+\frac{1}{2}+c \sqrt{h}\right|\right) \\
& \times\left(\left|\frac{1}{2} b h(1+a h)\right|+\left|\frac{1}{2} b h\right|\right. \\
& \left.+\left|\frac{1}{2}(1+a h)^{2}+\frac{1}{2}-c \sqrt{h}\right|\right)
\end{aligned}
$$

Since $\eta_{n}$ 's follow two-point distribution, we get $\left|X_{n}\right| \rightarrow$ 0 , a.s. $(n \rightarrow \infty)$ if $R_{T}(h, a, b, c)<1$, which means that the Heun method in (12) is T-stable.

Similarly, for the Heun method in (13), we can get $\widetilde{R}_{T}(h, a, b, c, d)$ as follows:

$$
\begin{aligned}
\widetilde{R}_{T}(h, a, b, c, d) & \\
= & \left(\left|\frac{1}{2} b h(1+a h)+d \sqrt{h}\right|+\left|\frac{1}{2} b h\right|\right. \\
& \left.+\left|\frac{1}{2}(1+a h)^{2}+\frac{1}{2}+c \sqrt{h}\right|\right) \\
& \times\left(\left|\frac{1}{2} b h(1+a h)-d \sqrt{h}\right|+\left|\frac{1}{2} b h\right|\right. \\
& \left.+\left|\frac{1}{2}(1+a h)^{2}+\frac{1}{2}-c \sqrt{h}\right|\right),
\end{aligned}
$$

and $\left|X_{n}\right| \rightarrow 0$, a.s. $(n \rightarrow \infty)$ if $\widetilde{R}_{T}(h, a, b, c, d)<1$, which means that the Heun method in (13) is T-stable.

Theorem 5. Suppose (6) meets the condition in (9). The Heun method in (12) is T-stable if $h<H$, where

$$
H=\min \left\{\frac{2}{|b|-a}, \frac{1}{4 c^{2}}\right\} \text {. }
$$

Proof. The condition in (9) gives $a<0$. Denote

$$
\begin{aligned}
R_{1}(h, a, b, c)= & \left|\frac{1}{2} b h(1+a h)\right|+\left|\frac{1}{2} b h\right| \\
& +\left|\frac{1}{2}(1+a h)^{2}+\frac{1}{2}+c \sqrt{h}\right|, \\
R_{2}(h, a, b, c)= & \left|\frac{1}{2} b h(1+a h)\right|+\left|\frac{1}{2} b h\right| \\
& +\left|\frac{1}{2}(1+a h)^{2}+\frac{1}{2}-c \sqrt{h}\right|,
\end{aligned}
$$

and $R_{T}(h, a, b, c)=R_{1}(h, a, b, c) R_{2}(h, a, b, c)$.

For $b \neq 0$, we have $a+b<0, a-b<0$ from the condition in (9). Let

$$
h_{1}=-\frac{1}{a}, \quad h_{2}=\min \left\{-\frac{1}{a}, \frac{1}{4 c^{2}}\right\} \text {. }
$$

Only the conclusion of the theorem when $b<0, c>0$ is proven, and that of the theorem when $b<0, c<0$ or $b>$ $0, c>0$ or $b>0, c<0$ can be proven similarly.

If $h<h_{2}$, then $h<-1 / a$ and $h<1 / 4 c^{2}$, and we have $1+a h>0$ and $(1 / 2)-c \sqrt{h}>0$. Hence

$$
\begin{aligned}
R_{1}(h, a, b, c)+R_{2}(h, a, b, c) & \\
\quad & =-b h(1+a h)-b h+(1+a h)^{2}+1 \\
& =(a-b)(a h+2) h+2 .
\end{aligned}
$$

Since $a h+1>0$ and $a-b<0$, we have $a h+2>0$ and

$$
0<R_{1}(h, a, b, c)+R_{2}(h, a, b, c)<2 .
$$


Consequently,

$$
\begin{aligned}
R_{T}(h, a, b, c) & =R_{1}(h, a, b, c) R_{2}(h, a, b, c) \\
& \leq\left[\frac{R_{1}(h, a, b, c)+R_{2}(h, a, b, c)}{2}\right]^{2}<1,
\end{aligned}
$$

which means that the Heun method (12) is T-stable if $h<h_{2}$.

(a) When $h_{1}=-1 / a<H$, if $H=1 / 4 c^{2}$, then $h_{2}=$ $\min \left\{-1 / a, 1 / 4 c^{2}\right\}=-1 / a=h_{1}$.

If $H=2 /(|b|-a)$, then $H \leq 1 / 4 c^{2}$, which yields $h_{1}=$ $-1 / a<H \leq 1 / 4 c^{2}$, and $h_{2}=-1 / a=h_{1}$. Hence $h_{2}=h_{1}$ if $h_{1}<H$.

For obtaining the result of this theorem in this case, the left work is to prove the method is T-stable for $h_{1} \leq h<H$. that is,

If $h_{1} \leq h<H$, then $-1 / a \leq h<1 / 4 c^{2}, h<2 /(|b|-a)$;

$$
1+a h \leq 0, \quad \frac{1}{2}-c \sqrt{h}>0, \quad(a-|b|) h+2>0 .
$$

Since $a-|b| \leq a+b$, we get $(a+b) h+2 \geq(a-|b|) h+2>0$. Since

$$
\begin{aligned}
R_{1}(h, a, b, c)+R_{2}(h, a, b, c) & \\
\quad & b h(1+a h)-b h+(1+a h)^{2}+1 \\
& =a h[(a+b) h+2]+2,
\end{aligned}
$$

we have $0<R_{1}(h, a, b, c)+R_{2}(h, a, b, c)<2$ from $a<0$ and $(*)$, and therefore

$$
\begin{aligned}
R_{T}(h, a, b, c) & =R_{1}(h, a, b, c) R_{2}(h, a, b, c) \\
& \leq\left[\frac{R_{1}(h, a, b, c)+R_{2}(h, a, b, c)}{2}\right]^{2}<1,
\end{aligned}
$$

which means that the Heun method (12) is T-stable if $h<H$.

(b) When $h_{1}=H$, it is easy to know $h_{2}=h_{1}$, and the Heun method (12) is T-stable if $h<h_{2}=H$.

(c) When $h_{1}=-1 / a>H$, if $H=1 / 4 c^{2}$, then $h_{2}=$ $\min \left\{-1 / a, 1 / 4 c^{2}\right\}=1 / 4 c^{2}=H$. If $H=2 /(|b|-a)$, then $-1 / a>2 /(|b|-a)$. Solving this gives $a>-|b|$, which contradicts the condition in (9). So $h_{2}=H$ if $h_{1}>H$. Hence the Heun method in (12) is T-stable if $h<h_{2}=H$.

The discussion above shows that the Heun method in (12) is T-stable provided that $h<H$, which completes the proof.

Theorem 6. Suppose (7) meets the condition in (10). The Heun method in (13) is T-stable if $H_{1}<h<H_{2}$, where

$$
H_{1}=\frac{4 d^{2}}{(a+|b|)^{2}}, \quad H_{2}=\min \left\{-\frac{1}{a}, \frac{1}{4 c^{2}}, \frac{4 d^{2}}{b^{2}}\right\} .
$$

Proof. The condition in (10) gives $a<0$. Denote

$$
\begin{aligned}
\widetilde{R}_{1}(h, a, b, c, d)= & \left|\frac{1}{2} b h(1+a h)+d \sqrt{h}\right| \\
& +\left|\frac{1}{2} b h\right|+\left|\frac{1}{2}(1+a h)^{2}+\frac{1}{2}+c \sqrt{h}\right|, \\
\widetilde{R}_{2}(h, a, b, c, d)= & \left|\frac{1}{2} b h(1+a h)-d \sqrt{h}\right| \\
& +\left|\frac{1}{2} b h\right|+\left|\frac{1}{2}(1+a h)^{2}+\frac{1}{2}-c \sqrt{h}\right|,
\end{aligned}
$$

and then $\widetilde{R}_{T}(h, a, b, c, d)=\widetilde{R}_{1}(h, a, b, c, d) \widetilde{R}_{2}(h, a, b, c, d)$ from (17).

(a) Suppose $b<0, c>0, d>0$, and $H_{1}<H_{2}$. With $h<H_{2}$, we have

$$
1+a h>0, \quad \frac{1}{2} b h+d \sqrt{h}>0, \quad \frac{1}{2}-c \sqrt{h}>0,
$$

and $\widetilde{R}_{1}(h, a, b, c, d)+\widetilde{R}_{2}(h, a, b, c, d)=(2 a-b) h+2 d \sqrt{h}+$ $a^{2} h^{2}+2$ from $(* *)$. With $h>H_{1}$, we have $h>4 d^{2} /(a-b)^{2}$; that is, $(2 a-b) h+2 d \sqrt{h}<a h$. The inequality $1+a h>0$ with $a<0$ gives $a h<-a^{2} h^{2}$, and therefore $(2 a-b) h+2 d \sqrt{h}<$ $-a^{2} h^{2}$. With this and $(* *)$, we get $0<\widetilde{R}_{1}(h, a, b, c, d)+$ $\widetilde{R}_{2}(h, a, b, c, d)<2$. Consequently,

$$
\begin{aligned}
\widetilde{R}_{T}(h, a, b, c, d) & =\widetilde{R}_{1}(h, a, b, c, d) \widetilde{R}_{2}(h, a, b, c, d) \\
& <\left[\frac{\widetilde{R}_{1}(h, a, b, c, d)+\widetilde{R}_{2}(h, a, b, c, d)}{2}\right]^{2}<1,
\end{aligned}
$$

which means the Heun method in (13) is T-stable.

In the same way, we can prove that the Heun method in (13) is T-stable when $b<0, c>0, d<0$ or $b<0, c<0, d>0$ or $b<0, c<0, d<0$.

(b) Suppose $b>0, c>0, d>0$, and $H_{1}<H_{2}$. With $h<H_{2}$, we get

$$
1+a h>0, \quad \frac{1}{2} b h-d \sqrt{h}<0, \quad \frac{1}{2}-c \sqrt{h}>0,
$$

and $\widetilde{R}_{1}(h, a, b, c, d)+\widetilde{R}_{2}(h, a, b, c, d)=(2 a+b) h+2 d \sqrt{h}+$ $a^{2} h^{2}+2$ from $(* *)$. With $h>H_{1}$, we have $h>4 d^{2} /(a+b)^{2}$; that is, $(2 a+b) h+2 d \sqrt{h}<a h$. The inequality $1+a h>0$ with $a<0$ gives $a h<-a^{2} h^{2}$, and therefore $(2 a+b) h+2 d \sqrt{h}<$ $-a^{2} h^{2}$. With this and $(* *)$, we get $0<\widetilde{R}_{1}(h, a, b, c, d)+$ $\widetilde{R}_{2}(h, a, b, c, d)<2$. Consequently,

$$
\begin{aligned}
\widetilde{R}_{T}(h, a, b, c, d) & =\widetilde{R}_{1}(h, a, b, c, d) \widetilde{R}_{2}(h, a, b, c, d) \\
& <\left[\frac{\widetilde{R}_{1}(h, a, b, c, d)+\widetilde{R}_{2}(h, a, b, c, d)}{2}\right]^{2}<1,
\end{aligned}
$$

which means that the Heun method in (13) is T-stable.

In the same way, we can prove that the Heun method in (13) is T-stable when $b>0, c>0, d<0$ or $b>0, c<0, d>0$ or $b>0, c<0, d<0$.

The proof of the theorem is complete. 


\section{T-Stability of the Balanced Method}

The balanced method applied to (6) and (7), respectively, gives

$$
\begin{aligned}
& X_{n+1}=\left(1+\frac{a h+c \Delta W_{n}}{1+C}\right) X_{n}+\left(\frac{b h}{1+C}\right) X_{n-m}, \\
& X_{n+1}=\left(1+\frac{a h+c \Delta W_{n}}{1+C}\right) X_{n}+\left(\frac{b h+d \Delta W_{n}}{1+C}\right) X_{n-m},
\end{aligned}
$$

where $C=C_{0} h+C_{1}\left|\Delta W_{n}\right|$ and $C_{0}$ and $C_{1}$ are real numbers. In the analysis of T-stability of balanced method, we also use two-point random variables for the driving process.

For the balanced method in (31), we have

$$
\begin{aligned}
\left|X_{n+1}\right| \leq\left(\left|1+\frac{a h+c \Delta W_{n}}{1+C_{0} h+C_{1}\left|\Delta W_{n}\right|}\right|\right. \\
\left.+\left|\frac{b h}{1+C_{0} h+C_{1}\left|\Delta W_{n}\right|}\right|\right) \max \left\{\left|X_{n}\right|,\left|X_{n-m}\right|\right\} .
\end{aligned}
$$

Denote

$$
\begin{aligned}
R(h, a, b, c)= & \left|1+\frac{a h+c \eta_{n} \sqrt{h}}{1+C_{0} h+C_{1}\left|\eta_{n}\right| \sqrt{h}}\right| \\
& +\left|\frac{b h}{1+C_{0} h+C_{1}\left|\eta_{n}\right| \sqrt{h}}\right|, \\
R_{T}(h, a, b, c)= & \left(\left|1+\frac{a h+c \sqrt{h}}{1+C_{0} h+C_{1} \sqrt{h}}\right|\right. \\
& \left.+\left|\frac{b h}{1+C_{0} h+C_{1} \sqrt{h}}\right|\right) \\
& \times\left(\left|1+\frac{a h-c \sqrt{h}}{1+C_{0} h+C_{1} \sqrt{h}}\right|\right. \\
& \left.+\left|\frac{b h}{1+C_{0} h+C_{1} \sqrt{h}}\right|\right) .
\end{aligned}
$$

The discussion about the Heun method in Section 3 implies $\left|X_{n}\right| \rightarrow 0$, a.s. $(n \rightarrow \infty)$ if $R_{T}(h, a, b, c)<1$, which means that the balanced method in (31) is T-stable if $R_{T}(h, a, b, c)<$ 1 .

Similarly, for the balanced method in (32), we get

$$
\begin{aligned}
\widetilde{R}_{T}(h, a, b, c, d) & \left(\left|1+\frac{a h+c \sqrt{h}}{1+C_{0} h+C_{1} \sqrt{h}}\right|+\left|\frac{b h+d \sqrt{h}}{1+C_{0} h+C_{1} \sqrt{h}}\right|\right) \\
& \times\left(\left|1+\frac{a h-c \sqrt{h}}{1+C_{0} h+C_{1} \sqrt{h}}\right|+\left|\frac{b h-d \sqrt{h}}{1+C_{0} h+C_{1} \sqrt{h}}\right|\right),
\end{aligned}
$$

and $\left|X_{n}\right| \rightarrow$ 0, a.s. $(n \rightarrow \infty)$ if $\widetilde{R}_{T}(h, a, b, c, d)<1$, which means that the Heun method in (32) is T-stable if $\widetilde{R}_{T}(h, a, b, c, d)<1$.
Theorem 7. Suppose (6) meets the condition in (9). The balanced method in (31) is T-stable if $F(h) \geq 0$, where

$$
F(h)=\left(C_{0}+a\right) h+\left(C_{1}-|c|\right) \sqrt{h} .
$$

Proof. The condition in (9) gives $a<0$. Denote

$$
\begin{aligned}
R_{1}(h, a, b, c)= & \left|1+\frac{a h+c \sqrt{h}}{1+C_{0} h+C_{1} \sqrt{h}}\right| \\
& +\left|\frac{b h}{1+C_{0} h+C_{1} \sqrt{h}}\right|, \\
R_{2}(h, a, b, c)= & \left|1+\frac{a h-c \sqrt{h}}{1+C_{0} h+C_{1} \sqrt{h}}\right| \\
& +\left|\frac{b h}{1+C_{0} h+C_{1} \sqrt{h}}\right|,
\end{aligned}
$$

and $R_{T}(h, a, b, c)=R_{1}(h, a, b, c) R_{2}(h, a, b, c)$ from (34). It is easy to know $a+b<0, a-b<0$ for $b \neq 0$.

If $c>0, b>0$, we have

$$
\begin{aligned}
& C_{0} h+C_{1} \sqrt{h}+a h-c \sqrt{h} \geq 0, \\
& C_{0} h+C_{1} \sqrt{h}+a h+c \sqrt{h} \geq 0,
\end{aligned}
$$

from the assumption $F(h) \geq 0$, which applies $1+C_{0} h+$ $C_{1} \sqrt{h}>0$. Then $R_{1}(h, a, b, c)$ and $R_{2}(h, a, b, c)$ in $(* * *)$ become

$$
\begin{aligned}
& R_{1}(h, a, b, c)=\frac{1+C_{0} h+C_{1} \sqrt{h}+a h+c \sqrt{h}+b h}{1+C_{0} h+C_{1} \sqrt{h}}, \\
& R_{2}(h, a, b, c)=\frac{1+C_{0} h+C_{1} \sqrt{h}+a h-c \sqrt{h}+b h}{1+C_{0} h+C_{1} \sqrt{h}}, \\
& R_{1}(h, a, b, c)+R_{2}(h, a, b, c)=2+\frac{2(a+b) h}{1+C_{0} h+C_{1} \sqrt{h}} .
\end{aligned}
$$

Since $a+b<0, \quad 1+C_{0} h+C_{1} \sqrt{h}>0$, we have $0<$ $R_{1}(h, a, b, c)+R_{2}(h, a, b, c)<2$ and

$$
\begin{aligned}
R_{T}(h, a, b, c) & =R_{1}(h, a, b, c) R_{2}(h, a, b, c) \\
& <\left[\frac{R_{1}(h, a, b, c)+R_{2}(h, a, b, c)}{2}\right]^{2}<1,
\end{aligned}
$$

which means that balanced method in (31) is T-stable.

In the same way, we can prove that the balanced method in (31) is T-stable when

$$
\begin{gathered}
c>0, \quad b<0 \quad \text { or } c<0, \quad b>0, \\
\text { or } c<0, \quad b<0 .
\end{gathered}
$$

The proof of the theorem is complete.

Theorem 8. Suppose (7) meets the condition in (10). The balanced method in (32) is T-stable if $h>H$ and $F(h) \geq 0$, where

$$
H=\frac{d^{2}}{b^{2}}, \quad F(h)=\left(C_{0}+a\right) h+\left(C_{1}-|c|\right) \sqrt{h} .
$$




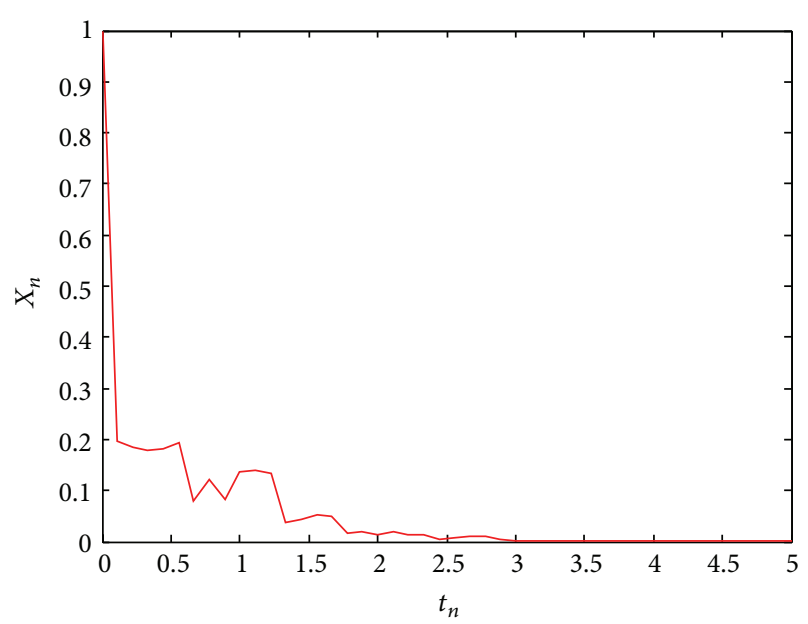

(a1)

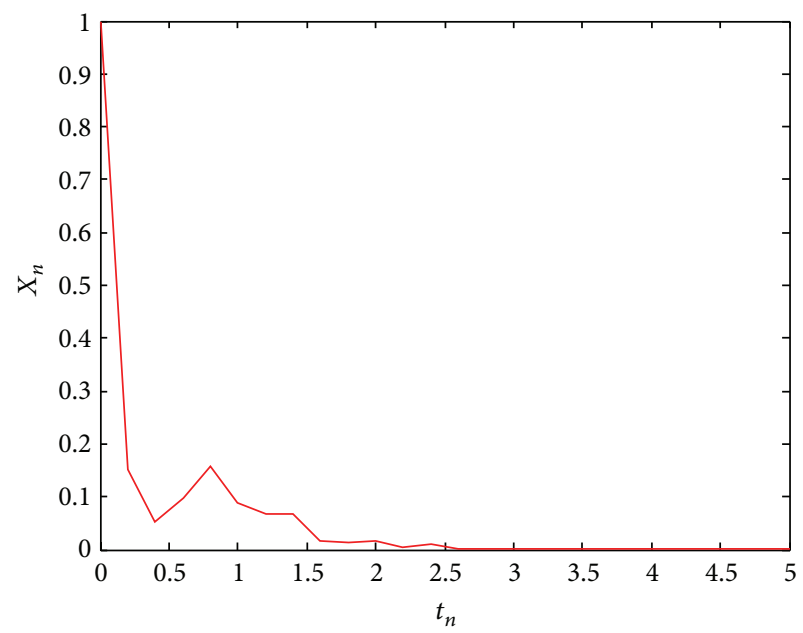

(c1)

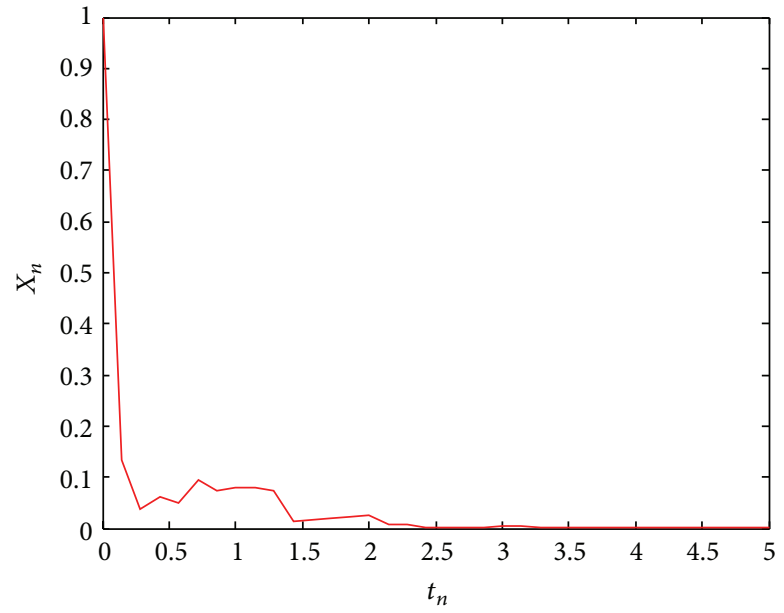

(b1)

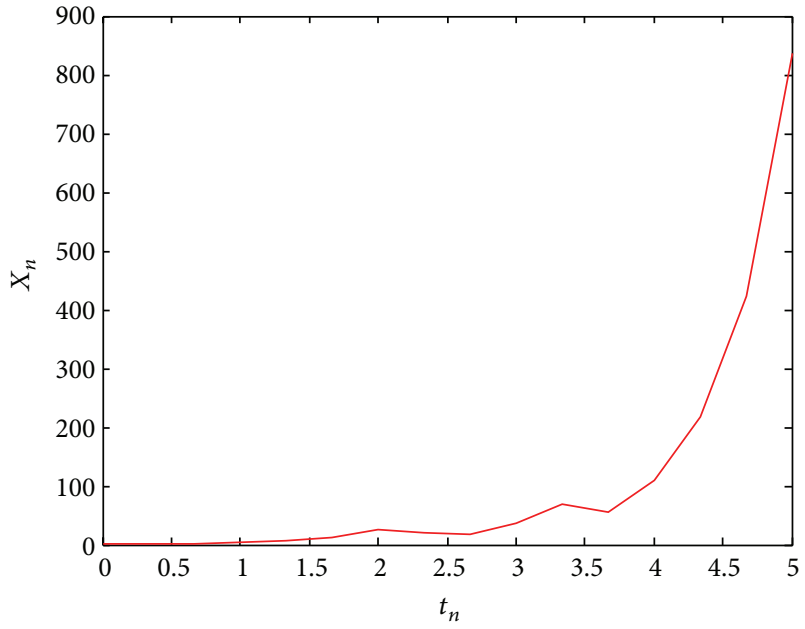

(d1)

FIGURE 1: Simulations with the Heun method for (6) with $a=-7, b=1$, and $c=1$. (a1) $h=\frac{1}{9}$, (b1) $h=\frac{1}{7},(\mathrm{c} 1) h=\frac{1}{5}$, and (d1) $h=1 / 3$.

Proof. The condition in (10) gives $a<0$. Denote

$$
\begin{aligned}
\widetilde{R}_{1}(h, a, b, c, d)= & \left|1+\frac{a h+c \sqrt{h}}{1+C_{0} h+C_{1} \sqrt{h}}\right| \\
& +\left|\frac{b h+d \sqrt{h}}{1+C_{0} h+C_{1} \sqrt{h}}\right|, \\
\widetilde{R}_{2}(h, a, b, c, d)= & \left|1+\frac{a h-c \sqrt{h}}{1+C_{0} h+C_{1} \sqrt{h}}\right| \\
& +\left|\frac{b h-d \sqrt{h}}{1+C_{0} h+C_{1} \sqrt{h}}\right|,
\end{aligned}
$$

and then $\widetilde{R}_{T}(h, a, b, c, d)=\widetilde{R}_{1}(h, a, b, c, d) \widetilde{R}_{2}(h, a, b, c, d)$ from (35). It is easy to know $a+b<0, a-b<0$ for $b \neq 0$.
If $c>0, b>0, d>0$, we have $b h-d \sqrt{h}>0$ from $h>H$ and

$$
\begin{aligned}
& C_{0} h+C_{1} \sqrt{h}+a h-c \sqrt{h} \geq 0, \\
& C_{0} h+C_{1} \sqrt{h}+a h+c \sqrt{h} \geq 0,
\end{aligned}
$$

from the assumption $F(h) \geq 0$, which yields $1+C_{0} h+$ $C_{1} \sqrt{h}>0$. Then $\widetilde{R}_{1}(h, a, b, c, d)$ and $\widetilde{R}_{2}(h, a, b, c, d)$ in $(* * * *)$ become

$$
\begin{gathered}
\widetilde{R}_{1}(h, a, b, c, d)=\frac{1+C_{0} h+C_{1} \sqrt{h}+a h+c \sqrt{h}+b h+d \sqrt{h}}{1+C_{0} h+C_{1} \sqrt{h}}, \\
\widetilde{R}_{2}(h, a, b, c, d)=\frac{1+C_{0} h+C_{1} \sqrt{h}+a h-c \sqrt{h}+b h-d \sqrt{h}}{1+C_{0} h+C_{1} \sqrt{h}}, \\
\widetilde{R}_{1}(h, a, b, c, d)+\widetilde{R}_{2}(h, a, b, c, d)=2+\frac{2(a+b) h}{1+C_{0} h+C_{1} \sqrt{h}} .
\end{gathered}
$$




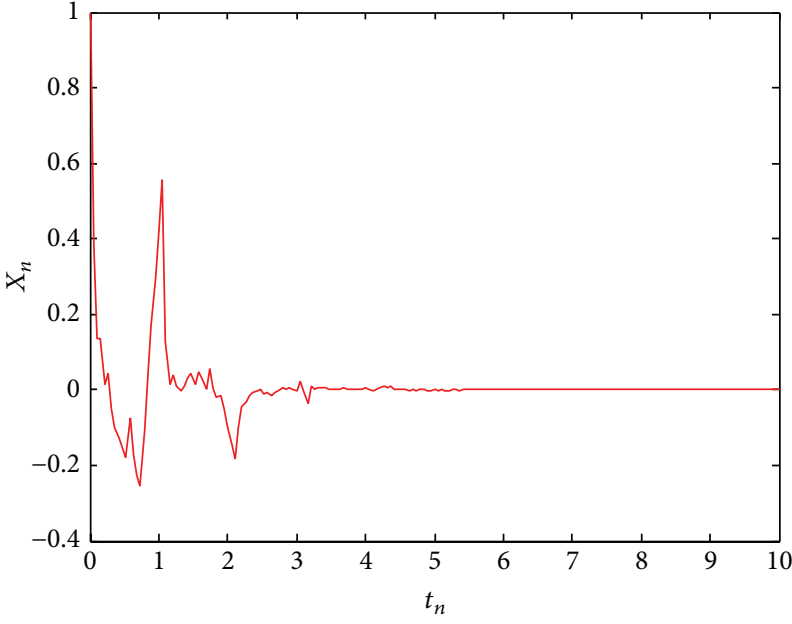

(a2)

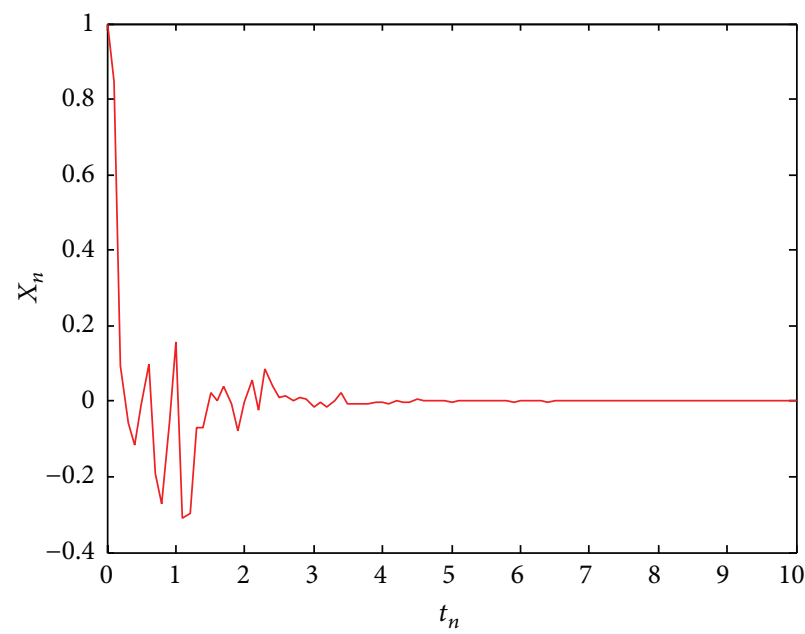

(c2)

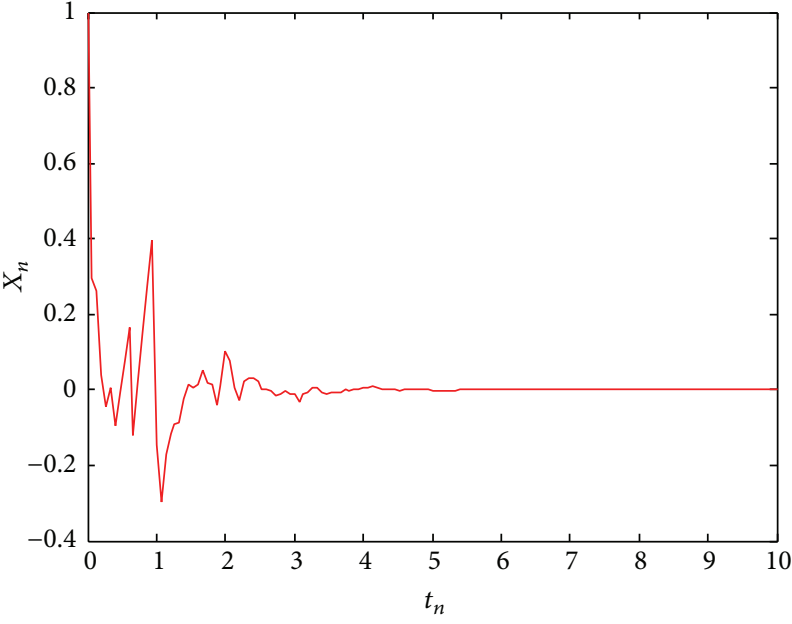

(b2)

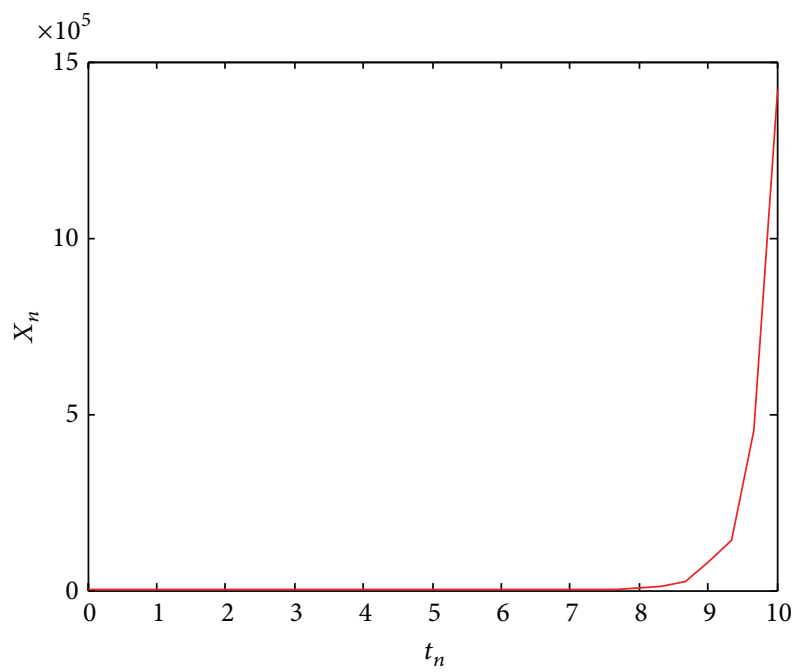

(d2)

FIGURE 2: Simulations with the Heun method for (7) with $a=-9, b=-1, c=1.1$, and $d=0.9$. (a2) $h=1 / 19,(\mathrm{~b} 2) h=1 / 15$, (c2) $h=1 / 11$, and (d2) $h=1 / 3$.

Since $a+b<0,1+C_{0} h+C_{1} \sqrt{h}>0$, we have $0<$ $\widetilde{R}_{1}(h, a, b, c, d)+\widetilde{R}_{2}(h, a, b, c, d)<2$, and $\widetilde{R}_{T}(h, a, b, c, d)=$ $\widetilde{R}_{1}(h, a, b, c, d) \widetilde{R}_{2}(h, a, b, c, d)<1$, which means that the balanced method in (32) is T-stable.

In the same way, we can prove that the balanced method in (32) is T-stable when

$$
\begin{gathered}
c>0, \quad b>0, \quad d<0 \quad \text { or } \quad c>0, \quad b<0, \quad d>0 \\
\text { or } c>0, \quad b<0, \quad d<0 .
\end{gathered}
$$
and

If $c<0, b>0, d>0$, we get $b h-d \sqrt{h}>0$ from $h>H$

$$
\begin{aligned}
& C_{0} h+C_{1} \sqrt{h}+a h+c \sqrt{h} \geq 0, \\
& C_{0} h+C_{1} \sqrt{h}+a h-c \sqrt{h} \geq 0
\end{aligned}
$$

from the assumption $F(h) \geq 0$, which applies $1+C_{0} h+C_{1} \sqrt{h}>$ 0 . Then

$$
\widetilde{R}_{1}(h, a, b, c, d)+\widetilde{R}_{2}(h, a, b, c, d)=2+\frac{2(a+b) h}{1+C_{0} h+C_{1} \sqrt{h}}
$$

from $(* * * *)$. Since $a+b<0,1+C_{0} h+C_{1} \sqrt{h}>0$, we have

$$
0<\widetilde{R}_{1}(h, a, b, c, d)+\widetilde{R}_{2}(h, a, b, c, d)<2 ;
$$

consequently, $\widetilde{R}_{T}(h, a, b, c, d)<1$, which means that the balanced method in (32) is T-stable.

In the same way, we can prove that the balanced method in (32) is T-stable when

$$
\begin{gathered}
c<0, \quad b>0, \quad d<0 \quad \text { or } \quad c<0, \quad b<0, \quad d>0 \\
\text { or } c<0, \quad b<0, \quad d<0 .
\end{gathered}
$$

The proof of Theorem 8 is complete. 


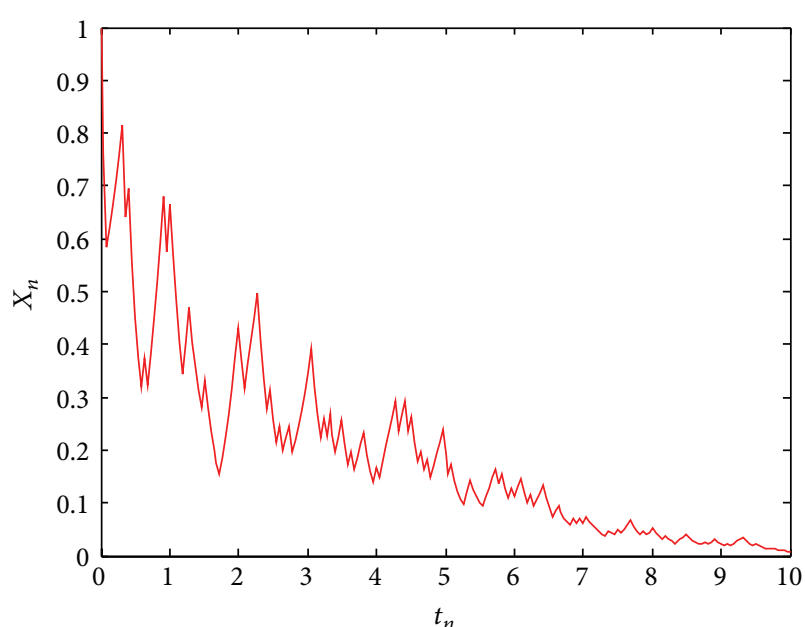

(a3)

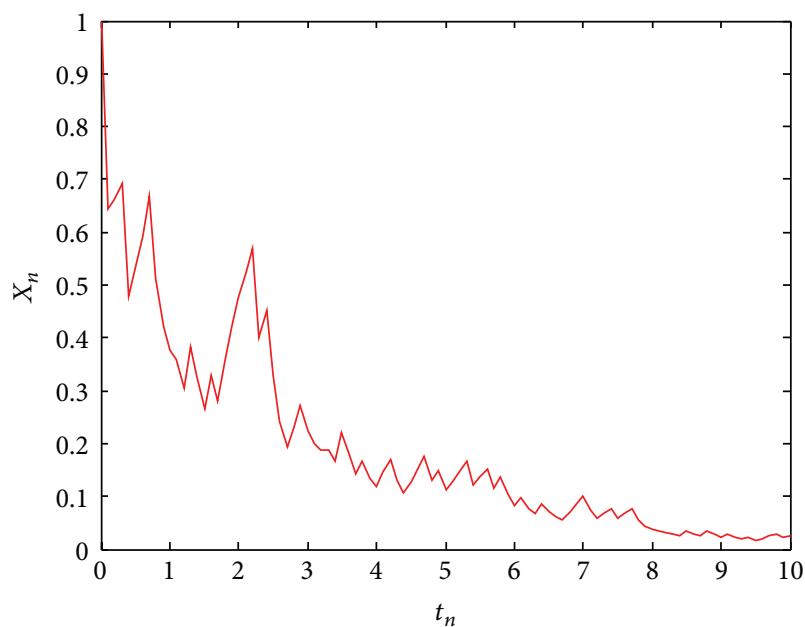

(c3)

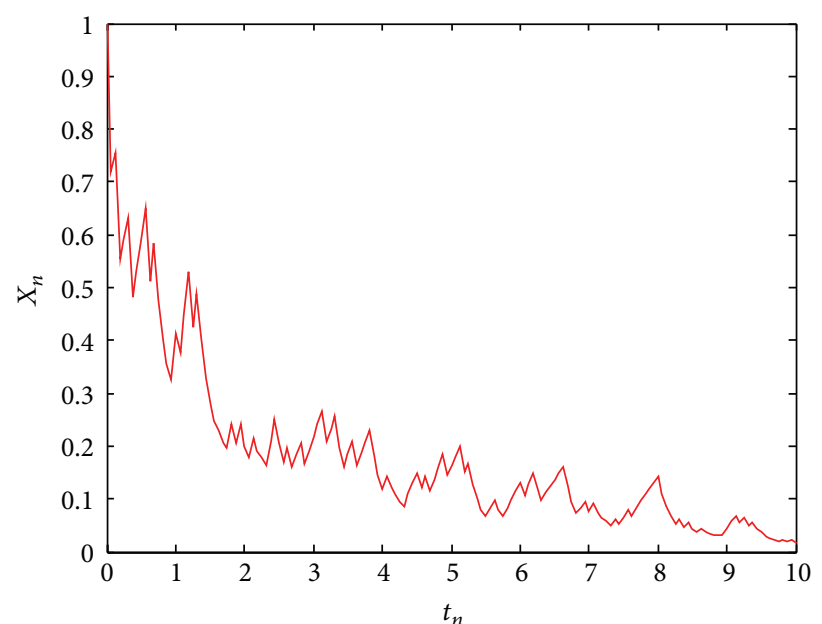

(b3)

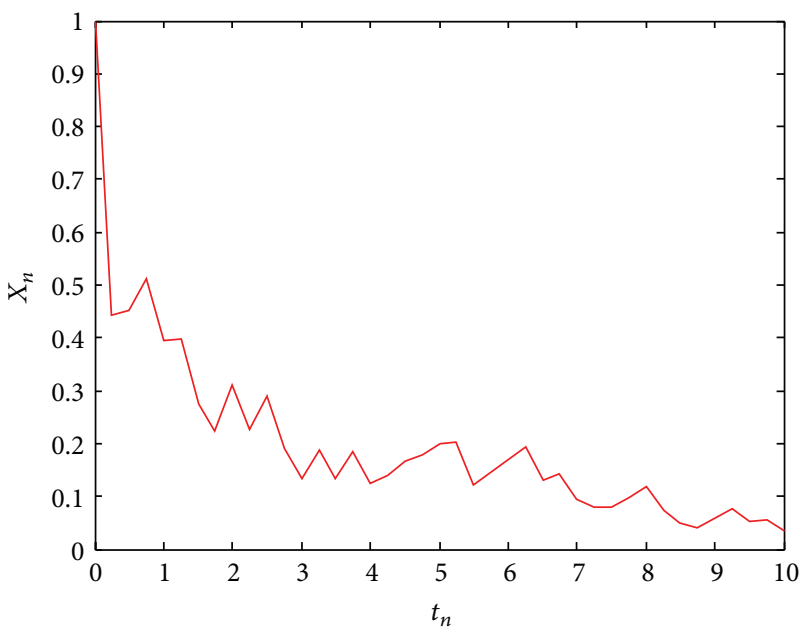

(d3)

Figure 3: Simulations with the balanced method for (6) with $a=-3, b=2$, and $c=1$. (a3) $h=1 / 22$, (b3) $h=1 / 16,(\mathrm{c} 3) h=1 / 10$, and (d3) $h=1 / 4$.

\section{Numerical Examples}

Consider test equations (6) and (7) with $\tau=1, \varphi(t)=t+$ $1, t \in[-1,0]$. In the following figures $t_{n}$ 's are nodes, and $X_{n}$ denotes the numerical solution at $t=t_{n}$.

Take $a=-7, b=1$, and $c=1$ in (6). From (18), we get $H=1 / 4$, which means that the Heun method in (12) is Tstable if $h<1 / 4$. Figure 1 shows that the Heun method in (12) is T-stable when $h=1 / 9, h=1 / 7$, and $h=1 / 5$ but is unstable when $h=1 / 3$, since $h=1 / 3$ exceeds the range of $h$ in Theorem 5 , which verifies Theorem 5 .

Take $a=-9, b=-1, c=1.1$, and $d=0.9$ in (7). From (26), we get $H_{1} \approx 1 / 20, H_{2}=1 / 9$, which means that the Heun method in (13) is T-stable if $1 / 20<h<1 / 9$. Figure 2 shows that the Heun method in (13) is T-stable when $h=1 / 19, h=1 / 15$, and $h=1 / 11$, but it is unstable when $h=1 / 3$, since $h=1 / 3$ exceeds the range of $h$ in Theorem 6 , which verifies Theorem 6 .
Take $a=-3, b=2$, and $c=1$ in (6) and $C_{0}=1, C_{1}=2$ in the balanced method in (31). Then the balanced method in (31) is T-stable if $h \leq 1 / 4$ from Theorem 7 . Figure 3 shows that the balanced method in (31) is T-stable when $h=1 / 22$, $h=1 / 16, h=1 / 10$, and $h=1 / 4$, since these $h$ 's are in the range of $h$ in Theorem 7 , which verifies Theorem 7 .

Take $a=-5, b=1, c=1$, and $d=0.2$ in (7) and $C_{0}=$ 2, $C_{1}=2$ in the balanced method (32). Then the balanced method in (32) is T-stable if $1 / 25<h \leq 1 / 9$ from Theorem 8 . Figure 4 shows that the balanced method in (32) is T-stable when $h=1 / 15, h=1 / 13, h=1 / 11$, and $h=1 / 9$, since these $h$ 's are in the range of $h$ in Theorem 8 , which verifies Theorem 8.

\section{Conclusion}

In this paper, T-stability of the Heun methods and the balanced methods for two kinds of linear SDDEs is studied. 


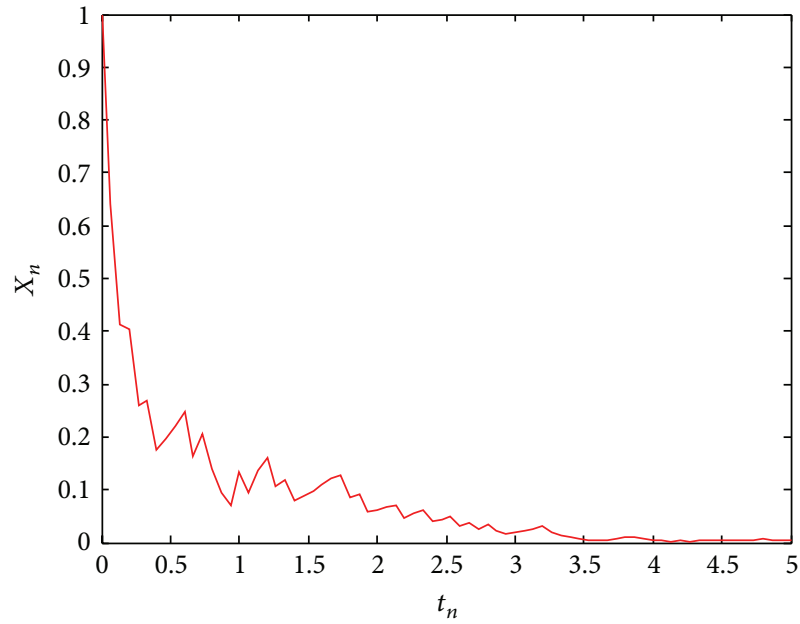

(a4)

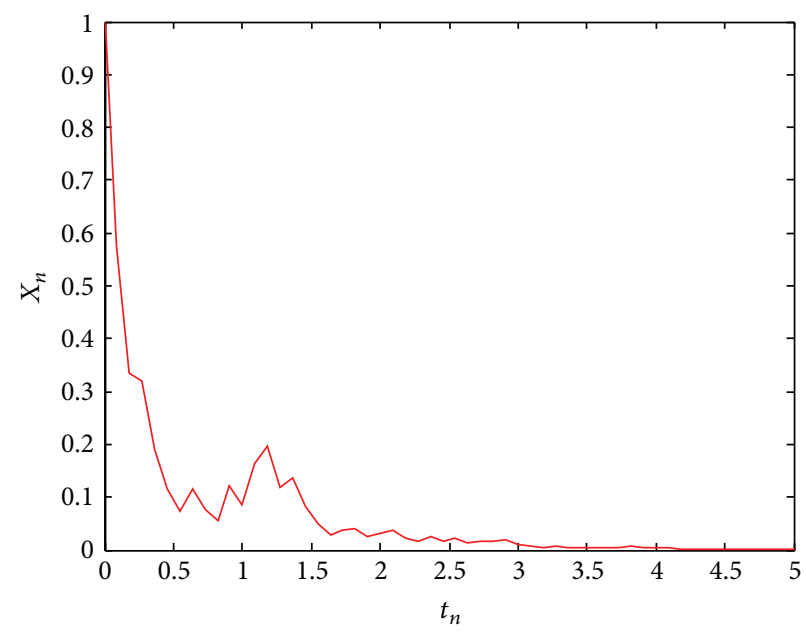

(c4)

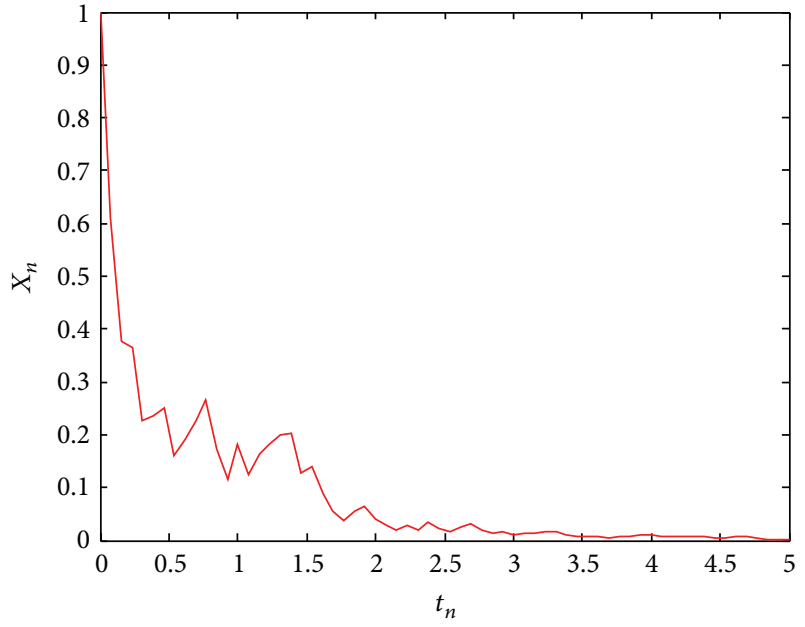

(b4)

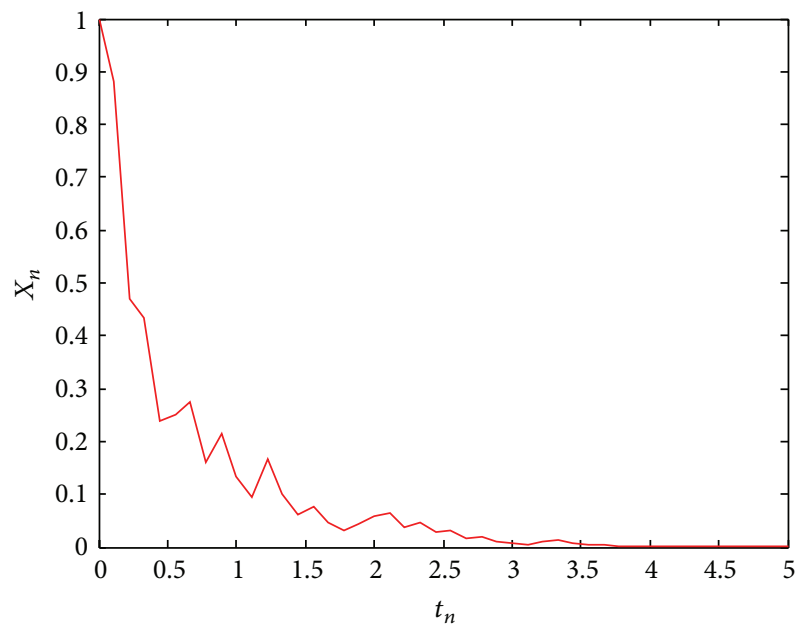

(d4)

FIGURE 4: Simulations with the balanced method for (7) with $a=-5, b=1, c=1$, and $d=0.2$. (a4) $h=1 / 15,(\mathrm{~b} 4) h=1 / 13,(\mathrm{c} 4) h=1 / 11$, and (d4) $h=1 / 9$.

The Wiener increment of the numerical methods in this paper is approximated by a discrete random variable with twopoint distribution in the process of the study of T-stability. The T-stable conditions for the Heun methods and balanced methods are given, respectively, and are verified by some numerical examples.

\section{Conflict of Interests}

The authors declare that there is no conflict of interests regarding the publication of this paper.

\section{Acknowledgments}

This paper is supported by the National Natural Science Foundation of China (no. 61272024) and the Anhui Provincial Natural Science Foundation (no. 11040606M06).

\section{References}

[1] W. Cao, M. Liu, and Z. Fan, "MS-stability of the EulerMaruyama method for stochastic differential delay equations," Applied Mathematics and Computation, vol. 159, no. 1, pp. 127$135,2004$.

[2] M. Liu, W. Cao, and Z. Fan, "Convergence and stability of the semi-implicit Euler method for a linear stochastic differential delay equation," Journal of Computational and Applied Mathematics, vol. 170, no. 2, pp. 255-268, 2004.

[3] Z. Wang and C. Zhang, "An analysis of stability of Milstein method for stochastic differential equations with delay," Computers \& Mathematics with Applications, vol. 51, no. 9-10, pp. 1445-1452, 2006.

[4] W. Wang and Y. Chen, "Mean-square stability of semi-implicit Euler method for nonlinear neutral stochastic delay differential equations," Applied Numerical Mathematics, vol. 61, no. 5, pp. 696-701, 2011.

[5] W. Q. Wang and Y. P. Chen, "Numerical stability of Heun methods for nonlinear stochastic delay differential equations," 
Mathematica Numerica Sinica, vol. 33, no. 1, pp. 69-76, 2011 (Chinese).

[6] Y. X. Tan, S. Q. Gan, and X. J. Wang, "Mean-square convergence and stability of a balanced method for stochastic delay differential equations," Mathematica Numerica Sinica, vol. 33, no. 1, pp. 25-36, 2011 (Chinese).

[7] Y. Saito and T. Mitsui, "T-stability of numerical scheme for stochastic differential equations," World Scientific Series in Applicable Analysis, vol. 2, pp. 333-344, 1993.

[8] K. Burrage and T. Tian, "A note on the stability properties of the Euler methods for solving stochastic differential equations," New Zealand Journal of Mathematics, vol. 29, p. 2000, 1999.

[9] K. Burrage, P. Burrage, and T. Mitsui, "Numerical solutions of stochastic differential equations-implementation and stability issues," Journal of Computational and Applied Mathematics, vol. 125, no. 1-2, pp. 171-182, 2000.

[10] W. Cao, "T-stability of the semi-implicit Euler method for delay differential equations with multiplicative noise," Applied Mathematics and Computation, vol. 216, no. 3, pp. 999-1006, 2010.

[11] A. Rathinasamy and K. Balachandran, "T-stability of the splitstep $\theta$-methods for linear stochastic delay integro-differential equations," Nonlinear Analysis: Hybrid Systems, vol. 5, no. 4, pp. 639-646, 2011.

[12] J.-H. Yang and M. Liu, "T-stability of the $\theta$-method for a stochastic pantograph differential equation," Journal of Mathematics, vol. 33, no. 1, pp. 27-34, 2013.

[13] J. Alcock and K. Burrage, "A note on the balanced method," BIT Numerical Mathematics, vol. 46, no. 4, pp. 689-710, 2006.

[14] L. Hu and S. Gan, "Convergence and stability of the balanced methods for stochastic differential equations with jumps," International Journal of Computer Mathematics, vol. 88, no. 10, pp. 2089-2108, 2011.

[15] X. Mao, "Razumikhin-type theorems on exponential stability of stochastic functional-differential equations," Stochastic Processes and Their Applications, vol. 65, no. 2, pp. 233-250, 1996. 


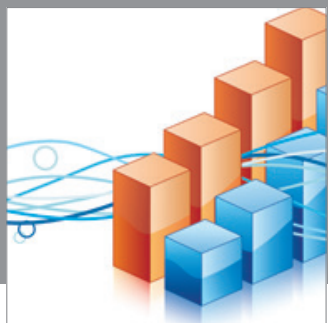

Advances in

Operations Research

mansans

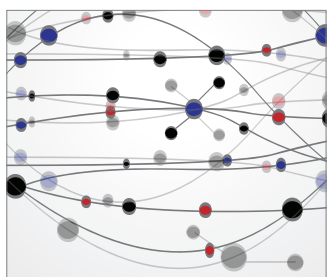

The Scientific World Journal
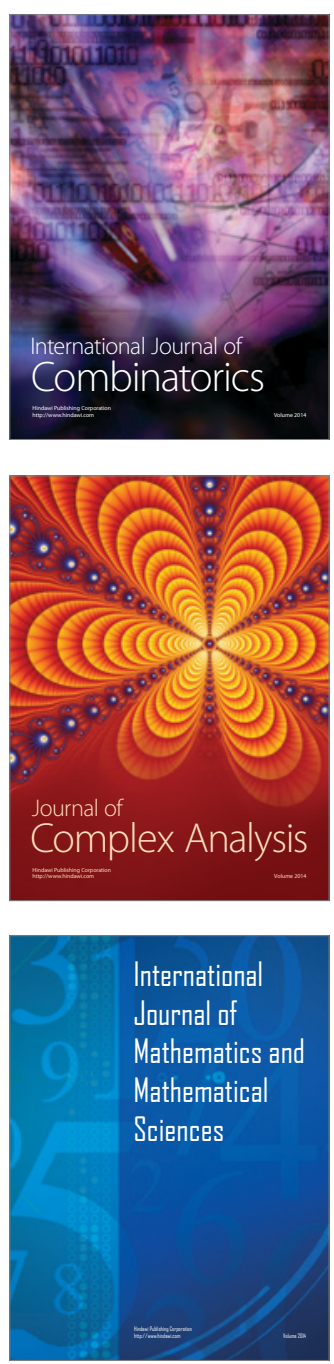
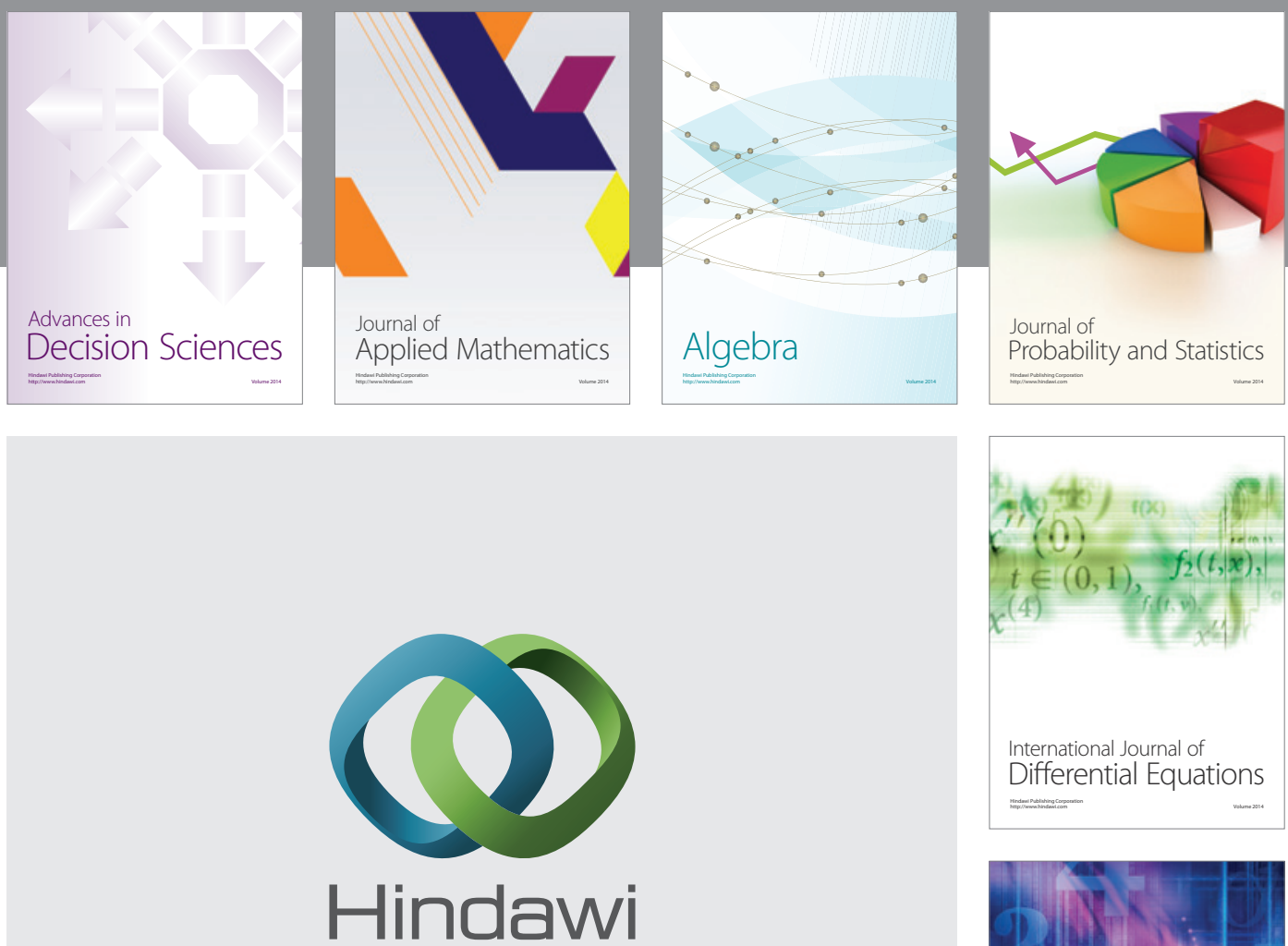

Submit your manuscripts at http://www.hindawi.com
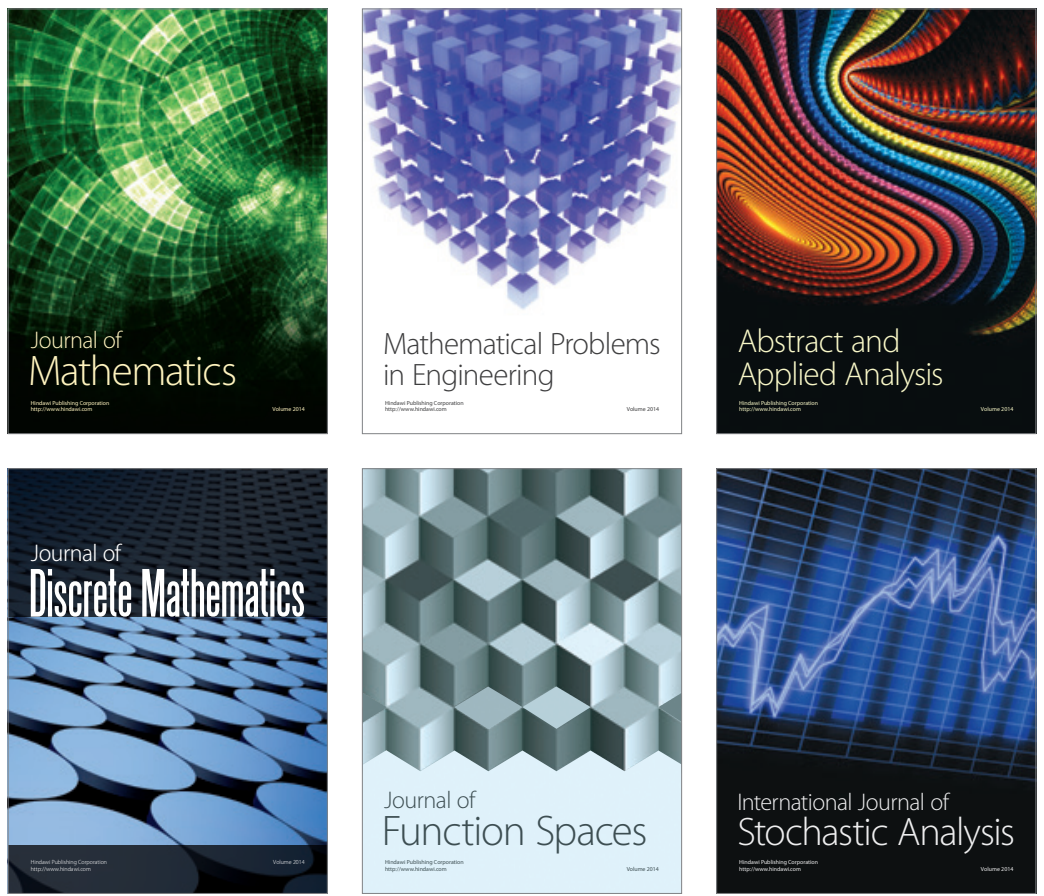

Journal of

Function Spaces

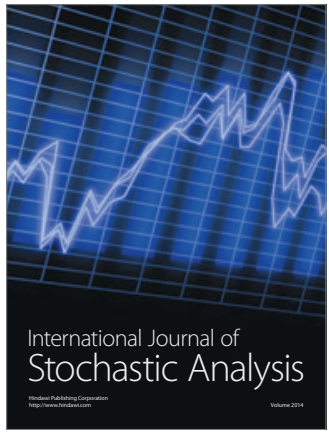

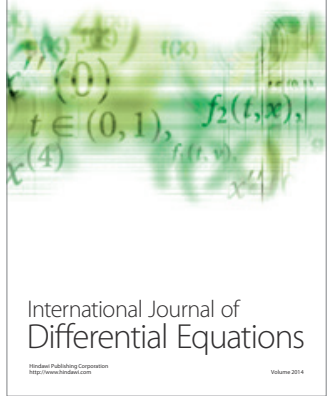
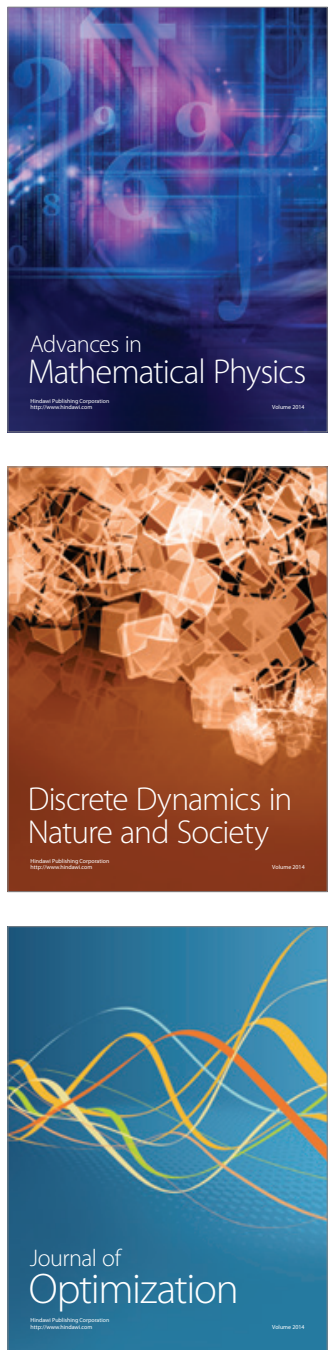